\title{
Possibility of the existence of charmed exotica
}

\author{
Hyun-Chul Kim, ${ }^{1,2}$ Maxim V. Polyakov, ${ }^{3,4}$ and Michał Praszałowicz ${ }^{5}$ \\ ${ }^{1}$ Department of Physics, Inha University, Incheon 22212, Republic of Kored* \\ ${ }^{2}$ School of Physics, Korea Institute for Advanced Study (KIAS), Seoul 02455, Republic of Korea \\ ${ }^{3}$ Institut für Theoretische Physik II, Ruhr-Universität Bochum, D-44780, Bochum, German $\chi^{\dagger}$ \\ ${ }^{4}$ Petersburg Nuclear Physics Institute, Gatchina, St. Petersburg 188 300, Russia \\ ${ }^{5}$ M. Smoluchowski Institute of Physics, Jagiellonian University, Eojasiewicza 11, 30-348 Kraków, Polan $q^{7}$
}

\begin{abstract}
We employ the chiral quark-soliton model to describe excited baryons with one heavy quark. Identifying known charmed baryons with multiplets allowed by the model, we argue that apart from regular excitations of the ground state multiplets, some of recently reported by the $\mathrm{LHCb}$ collaboration narrow $\Omega_{c}^{0}$ states, may correspond to the exotic pentaquarks. This interpretation can be easily verified experimentally, since exotic $\Omega_{c}^{0}$ states - contrary to the regular excitations - form isospin triplets, rather than singlets.
\end{abstract}

PACS numbers: 12.39.Hg, 14.20.Lq, 14.20.Mr, 11.30.Qc

Keywords: Heavy baryons, Mean field approach, Mass splittings of SU(3) baryons, Chiral soliton model, Flavor symmetry breaking, Exotic pentaquarks.

\section{INTRODUCTION}

In a very recent paper the LHCb collaboration announced five, or even six $\Omega_{c}^{0}$ states with masses in the range of $3-3.2 \mathrm{GeV}$ [1]. Naturally they correspond to the excitations of the ground state multiplets of charmed baryons that in this case form two $\mathrm{SU}(3)$ sextets: $1 / 2^{+}$ and $3 / 2^{+}$. In a recent paper 2 we have shown that these two sextets together with the ground state $\overline{\mathbf{3}}$ that comprises $\Lambda_{c}(2280)$ and $\Xi_{c}(2470)$ can be successfully described in terms of the chiral quark-soliton model ( $\chi$ QSM $)$ supplemented by an interaction with a heavy quark in such a way that heavy quark symmetry [3] is respected. A great advantage of the $\chi \mathrm{QSM}$ consists in a rather restrictive mass formula linking the spectra of light baryons with the heavy ones in question.

In the present paper we consider excitations of these ground state multiplets that are predicted within the $\chi$ QSM. They fall into two distinct categories: the regular excitations that correspond to one-particle excitation of the initial quark configuration and the exotic ones, which in the present work are identified with collective rotations of the soliton [4 6]. Since different assignments of the $\Omega_{c}^{0}$ states are possible, we propose criteria that have to be fulfilled by these excitations. In conclusion we argue that the most probable assignment is that $\Omega_{c}^{0}(3050)$ and $\Omega_{c}^{0}(3119)$ that are very narrow, with the decay widths around $1 \mathrm{MeV}$, correspond to the isospin triplet of pentaquarks in the $\mathrm{SU}(3) \overline{\mathbf{1 5}}$, while the remaining states, including rather wide bumps above $3.2 \mathrm{GeV}$, correspond to the quark excitations of the ground state sextets, and are therefore isospin singlets.

The LHCb discovery triggered several attempts to get an insight into the nature of the excited $\Omega_{c}^{0}$ 's in different

\footnotetext{
* hchkim@inha.ac.kr

$\dagger$ maxim.polyakov@tp2.ruhr-uni-bochum.de

$\ddagger$ michal.praszalowicz@uj.edu.pl
}

approaches. This includes the QCD sum rules [7] , the constituent quark models [10, and lattice QCD [11. In Refs. [12 14 the new states are treated as bound states of a charm quark and a light diquark, the authors of Ref. [15] viewed the new states as $\Xi_{c} K$ and $\Xi_{c}^{\prime} K$ molecular states and in some approaches [16] as pentaquarks.

The paper is organized as follows. First, we briefly describe the model and provide formulae for masses and discuss the decay widths (where possible). Next, we compare the $\chi$ QSM predictions with spectra of excited $\Lambda_{c}$ and $\Xi_{c}$, and then we discuss possible assignments of newly discovered $\Omega_{c}^{0}$ states within the pattern of mass splittings predicted by the model. Finally we conclude, and give estimates of masses of other members of $\overline{\mathbf{1 5}}$ and excited 6.

\section{CHIRAL QUARK-SOLITON MODEL FOR EXCITED HEAVY BARYONS}

The $\chi$ QSM is based on an argument of Witten 17 that in the limit of large number of colors, $N_{c}$ relativistic valence quarks generate chiral mean fields represented by a distortion of a Dirac sea that in turn influence the valence quarks themselves (for review see Ref.[18) forming a self-organized configuration called a soliton. Schematic pattern of light quark energy levels corresponding to this scenario is depicted in Fig. 1 1.a. Next, rotations of the soliton, both in flavor and configuration spaces, are quantized semiclassically and the collective Hamiltonian is computed. The model predicts rotational baryon spectra that satisfy the following selection rules:

- allowed $\mathrm{SU}(3)$ representations must contain states with hypercharge $Y^{\prime}=N_{c} / 3$,

- the isospin $\boldsymbol{T}^{\prime}$ of the states with $Y^{\prime}=N_{c} / 3$ couples with the soliton spin $\boldsymbol{J}$ to a singlet: $\boldsymbol{T}^{\prime}+\boldsymbol{J}=0$.

In the case of light parity $(+)$ baryons the lowest allowed representations are 8 of spin $1 / 2,10$ of spin $3 / 2$, 
and also exotic $\overline{\mathbf{1 0}}$ of spin $1 / 2$ with the lightest state corresponding to the putative $\Theta^{+}(1540)$.

In the recent paper 2 following [4] we have extended this model to baryons involving one heavy quark. In this case the valence level is occupied by $N_{c}-1$ light quarks (see Fig 1.b) that couple with a heavy quark $Q$ to form a color singlet. The first selection rule in this case reads: $Y^{\prime}=\left(N_{c}-1\right) / 3$. Therefore the lowest allowed SU(3) representations correspond to the soliton of spin 0 in $\overline{\mathbf{3}}$ and spin 1 in 6. Soliton spin couples with heavy quark spin to form spin $1 / 2 \mathrm{SU}(3)$ triplet and two sextets of spin $1 / 2$ and $3 / 2$ that are subject to a hyper-fine splitting. This pattern is confirmed by the data not only qualitatively but also quantitatively as shown in Ref. 22.

The next allowed representation of the rotational excitations corresponds to the exotic $\overline{\mathbf{1 5}}$ of spin 0 or spin 1 . As we will show below, the spin 1 soliton has a lower mass and when it couples with a heavy quark it forms spin $1 / 2$ or $3 / 2$ exotic multiplets that should be hyper-fine split similarly to the ground state sextets.

The rotational states described above do not change the parity of the ground state soliton and therefore they correspond to positive parity. In the present approach negative parity states are generated by soliton config- urations with one light valence quark excited from the valence level or from the Dirac sea. In this way one can successfully describe the light baryon spectrum up to 2 $\mathrm{GeV}$ [6]. In this case the second selection rule above is modified: $\boldsymbol{T}^{\prime}+\boldsymbol{J}=\boldsymbol{K}$, where $\boldsymbol{K}$ denotes so called grand spin of the excited valence quark. Let us remind that the energy levels of the Dirac operator in the presence of the chiral field with hedgehog symmetry are classified by an integer $K^{P}$ where $\boldsymbol{K}=\boldsymbol{l}+\boldsymbol{s}+\boldsymbol{t}$ with $\boldsymbol{l}$ standing for quark angular momentum, $\boldsymbol{s}$ for its spin and $\boldsymbol{t}$ for isospin [18. $P$ denotes parity. The soliton configuration with an excited quark develops its own rotational band. The selection rules for excited quark solitons can be therefore summarized as follows:

- allowed $\mathrm{SU}(3)$ representations must contain states with hypercharge $Y^{\prime}=\left(N_{c}-1\right) / 3$,

- the isospin $\boldsymbol{T}^{\prime}$ of the states with $Y^{\prime}=\left(N_{c}-1\right) / 3$ couples with the soliton spin $\boldsymbol{J}$ as follows: $\boldsymbol{T}^{\prime}+\boldsymbol{J}=$ $\boldsymbol{K}$, where $\boldsymbol{K}$ is the grand spin of the excited level.

The formula for the soliton mass in the chiral limit for the states in the $\mathrm{SU}(3)$ representation $\mathcal{R}$ has been derived in Ref. [5] and reads:

$\mathcal{M}^{(K)}=M_{\mathrm{sol}}^{(K)}+\frac{1}{2 I_{2}}\left[C_{2}(\mathcal{R})-T^{\prime}\left(T^{\prime}+1\right)-\frac{3}{4} Y^{\prime 2}\right]+\frac{1}{2 I_{1}}\left[\left(1-a_{K}\right) T^{\prime}\left(T^{\prime}+1\right)+a_{K} J(J+1)-a_{K}\left(1-a_{K}\right) K(K+1)\right]$

where $C_{2}(\mathcal{R})$ stands for the $\mathrm{SU}(3)$ Casimir operator. $M_{\mathrm{sol}}^{(K)} \sim N_{c}$ denotes classical soliton mass, $I_{1,2}$ represent moments of inertia and $a_{K}$ is a parameter that takes into account one-quark excitation. Although all these parameters can be in principle calculated in a specific model, we shall follow here a so called model-independent approach introduced in the context of the Skyrme model in Ref. [19, where all parameters are extracted from the experimental data.

Note that $a_{K}=0$ if all valence quarks occupy the ground state level and the soliton spin $J=T^{\prime}$. For solitons constructed from an excited valence quark configuration $a_{K} \neq 0$ and the soliton spin $J$ takes the following values:

$$
J=\left|T^{\prime}-K\right|, \ldots,\left|T^{\prime}+K\right| .
$$

In the case when the strange quark mass $m_{s}>m_{u, d} \simeq$ 0 , the soliton mass (1) has to be supplemented by the chiral symmetry breaking Hamiltonian [5]:

$$
H_{\mathrm{br}}=\alpha D_{88}^{(8)}+\beta \hat{Y}+\frac{\gamma}{\sqrt{3}} \sum_{i=1}^{3} D_{8 i}^{(8)} \hat{T}_{i}^{\prime}+\frac{\delta}{\sqrt{3}} \sum_{i=1}^{3} D_{8 i}^{(8)} \hat{K}_{i},
$$

which has to be evaluated between the collective wave functions [5, 6] that depend on the flavor rotation matrix $A$

$$
\begin{aligned}
\Psi_{\left(\mathcal{R}^{*} ;-Y^{\prime} T^{\prime} T_{3}^{\prime}\right)}^{\left(\mathcal{R} ; Y T T_{3}\right)} & =\sqrt{\operatorname{dim}(\mathcal{R})}(-)^{T_{3}^{\prime}-Y^{\prime} / 2} \\
& \times D_{\left(Y, T, T_{3}\right)\left(Y^{\prime}, T^{\prime},-T_{3}^{\prime}\right)}^{(\mathcal{R}) *}(A)
\end{aligned}
$$

coupled to the spin rotational wave function that depends on the rotational matrix $S$ and to the excited quark function $\chi_{K_{3}}$ :

$$
\begin{aligned}
& \Phi_{B, J, J_{3},\left(T^{\prime}, K\right)}^{(\mathcal{R})}=\sqrt{\frac{2 J+1}{2 K+1}} \sum_{T_{3}^{\prime}, J_{3}^{\prime}, K_{3}^{\prime}}\left(\begin{array}{cc|c}
T^{\prime} & J & K \\
-T_{3}^{\prime} & J_{3}^{\prime} & K_{3}^{\prime}
\end{array}\right) \\
& \times(-)^{-\left(T^{\prime}+T_{3}^{\prime}\right)} \Psi_{\left(\mathcal{R}^{*} ;-Y^{\prime} T^{\prime} T_{3}^{\prime}\right)}^{(\mathcal{R} ; A) D_{J_{3}^{\prime} J_{3}}^{(J) *}(S) \chi_{K_{3}^{\prime}},}
\end{aligned}
$$

where index $\left(\mathcal{R} ; Y T T_{3}\right)$ corresponds to the $\mathrm{SU}(3)$ quantum numbers of a given baryon in represntation $\mathcal{R}$, spin index $\left(\mathcal{R}^{*} ;-Y^{\prime} T^{\prime} T_{3}^{\prime}\right)$ is confined to a fixed value of $Y^{\prime}$ and formally transforms as a member of a representation conjugated to $\mathcal{R}$. The functions $D^{(\mathcal{R})}$ and $D^{(J)}$ are the $\mathrm{SU}(3)$ and $\mathrm{SU}(2)$ Wigner matrices, respectively, and $\chi_{K_{3}^{\prime}}=\left|K^{\prime}, K_{3}^{\prime}\right\rangle . \mathcal{O}\left(m_{s}\right)$ parameters $\alpha, \beta, \gamma$ and $\delta$ are computable in terms of single quark wave functions of valence and sea quarks. Their explicit form can be found in e.g. Ref. [5]. 
In order to construct a heavy baryon in the present model we have to strip off one light quark from the valence level and quantize the soliton with a new constraint $Y^{\prime}=\left(N_{c}-1\right) / 3$. The pertinent light quark configuration is shown in Fig. 1 b. Such a soliton is coupled with a heavy quark to form a color singlet, and the collective Hamiltonian has to be supplemented by a hyper-fine interaction, which we parametrize as follows [2]:

$$
H_{\mathrm{hf}}=\frac{2}{3} \frac{\kappa}{m_{Q}} \boldsymbol{J} \cdot \boldsymbol{J}_{Q}
$$

where $\kappa$ is flavor-indepenent. The operators $\boldsymbol{J}$ and $\boldsymbol{J}_{Q}$ represent the spin operators for the soliton and the heavy quark, respectively.

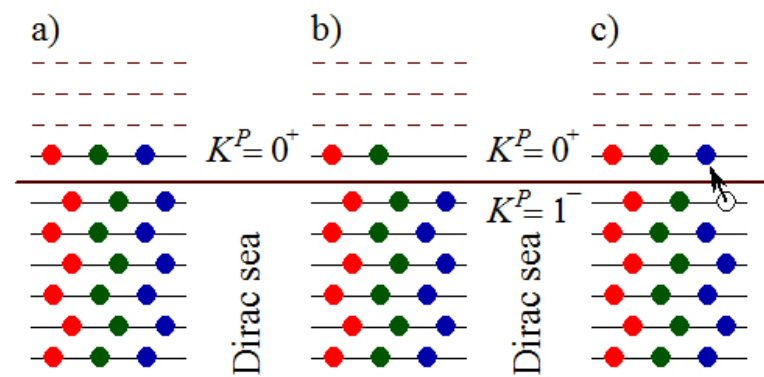

FIG. 1. Schematic pattern of light $(u$ and $d)$ quark levels in a self-consistent soliton configuration. In the left panel all sea levels are filled and $N_{c}$ ( $=3$ in the Figure) valence quarks occupy the $K^{P}=0^{+}$lowest positive energy level. Unoccupied positive energy levels are dpicted by dashed lines. In the middle panel one valence quark has been stripped off, and the soliton has to be supplemented by a heavy quark not shown in the Figure. In the right panel a possible excitation of a sea level quark, conjectured to be $K^{P}=1^{-}$, to the valence level is shown, and again the soliton has to couple to a heavy quark. Levels for strange quarks that exhibit different filling pattern are not shown.

\section{PHENOMENOLOGY OF HEAVY BARYONS IN $\chi$ QSM}

\section{A. Light sector phenomenology}

In order to estimate the heavy baryon masses in the $\chi$ QSM in the model-independent approach one fixes model parameters from the light sector and uses them for predictions in the heavy quark sector. This procedure, however, suffers from different systematic uncertainties. For example, there exist corrections to $M_{\text {sol }} \sim N_{c}$, that are of the order $\mathcal{O}\left(N_{c}^{0}\right)$ related to the Casimir energy [20, 21] and meson loops [22 25], which are beyond control in the present approach. Obviously, in a modelindependent approach these corrections are accommodated in $M_{\text {sol }}$ and also in $1 / I_{1,2}$. It is, however, unknown how they depend on the soliton quantum numbers and how they change in the presence of a heavy quark due to, for example, nontrivial color interactions between the soliton and an extra quark.

The splittings between multiplets are under much better control than than the absolute masses. For example, moment of inertia $I_{1}$ can be determined from the mass difference of the mean octet $\left(\mathcal{M}_{\mathbf{8}} \sim 1150 \mathrm{MeV}\right)$ and decuplet $\left(\mathcal{M}_{10} \sim 1380 \mathrm{MeV}\right)$ masses. Indeed, it follows from (1):

$$
\frac{1}{I_{1}}=\frac{2}{3}\left(\mathcal{M}_{\mathbf{1 0}}-\mathcal{M}_{\mathbf{8}}\right)=153 \mathrm{MeV},
$$

which agrees well with much more complete analysis of Ref. 26] giving $1 / I_{1}=160 \mathrm{MeV}$.

It is, however, much more difficult to estimate the second moment of inertia $I_{2}$, as it contributes only to the masses of exotic pentaquarks. Given the fact that the nonexotic members of $\overline{\mathbf{1 0}}$ can mix with regular baryons 27. $1 / I_{2}$ estimation suffers from large uncertainty. Also the mass of $\Theta^{+}$, whose existence is still upheld by the LEPS 28, 29, the DIANA [30] and a part of the CLAS experiment 31 (see, however, critique in Ref. [32]), suffers from an uncertainty of 20 $\mathrm{MeV}: 1520-1540 \mathrm{MeV}$. The best way to extract $1 / I_{2}$ is to use the mass of the exotic $\Xi_{5}$, since it does not mix with low mass regular hyperons. Using the values from Refs. 27, 33, we obtain:

$$
\frac{1}{I_{2}}=400-450 \mathrm{MeV}
$$

to be compared with even a larger estimate of Ref. [26]: $1 / I_{2}=470 \mathrm{MeV}$.

Splittings inside $\mathrm{SU}(3)$ multiplets are expressed in terms of $\mathcal{O}\left(m_{s}\right)$ parameters: $\alpha, \beta$ and $\gamma$. A rather detailed phenomenological analysis, which includes wave function corrections, isospin splittings and decay rates, yields rather well constrained result [26, which has been used in Ref. 2 and which we shall be using here as well:

$$
\alpha=-255 \mathrm{MeV}, \quad \beta=-140 \mathrm{MeV}, \quad \gamma=-101 \mathrm{MeV} .
$$

\section{B. Ground state multiplets}

In order to estimate the masses of the states in $\overline{\mathbf{3}}$ and 6 we have used the general formula (1) with one modification. Since the mean fields are generated by $N_{c}-1$ valence quarks (see Fig. 1. b), we have modified $\mathcal{O}\left(N_{c}\right)$ model parameters by the scaling factor $\rho=\left(N_{c}-1\right) / N_{c}$, namely: $I_{1,2} \rightarrow \rho I_{1,2}$ and $\alpha \rightarrow \bar{\alpha}=\rho \alpha$. This procedure has been applied in [2] both for average mass splittings between the multiplets and for $m_{s}$ splittings within multiplets of ground state baryons. While the rescaling works very well for $m_{s}$ splittings it is much less accurate for the moments of inertia $I_{1,2}$. Strictly speaking rescaling by a factor $\left(N_{c}-1\right) / N_{c}$ should work well only for quantities dominated by valence levels, which is probably not the case for $I_{1}$. Indeed, the rescaling factor that 
reproduces well $\mathbf{6}-\overline{\mathbf{3}}$ splitting is equal to $\rho=0.9$ rather than $2 / 3$.

Let us briefly summarize the results of Ref. [2]:

1. Lowest-lying heavy baryons can be indeed grouped in two $\mathrm{SU}(3)$ multiplets depicted in Fig. 2, an antitriplet of spin $1 / 2$ and two sextets of spin $1 / 2$ and $3 / 2$

2. The sextets are subject to the hyper-fine splitting (6) that scales like $1 / m_{Q}$ and the value of the splitting parameter for the charm quark is: $\kappa / m_{c}=70 \mathrm{MeV}$

3. Within each multiplet $\mathcal{R}$ isospin submultiplets split proportionally to the hypercharge: $\delta_{\mathcal{R}} Y$ with parameters $\delta_{\overline{\mathbf{3}}}=-180 \mathrm{MeV}$ and $\delta_{\mathbf{6}}=-120 \mathrm{MeV}$. These values, extracted from the heavy baryon data, are the same for $b$ and $c$ baryons, they are, however, lower by $11 \%$ than the values obtained from the splittings of the light baryon octet and decuplet with the help of Eq. (9). This can be explained by an $11 \%$ reduction of the strange quark mass in the presence of a heavy quark $Q$, since the ratio $\delta_{\overline{\mathbf{3}}} / \delta_{\mathbf{6}}$ is the same for both determinations.

4. Splittings between average $\overline{\mathbf{3}}$ and $\mathbf{6}$ masses are proportional to $1 / I_{1}$ and are equal in charm and bottom sectors. The value of $1 / I_{1}$ extracted from heavy baryon spectra and from the light baryon spectra require tiny rescaling factor $\rho=0.9$.

5. The model predicts a sum rule that links particles from different multiplets and allows to calculate $\Omega_{Q}^{*}$ mass, which is very well satisfied for $Q=c$ and gives a prediction for yet unmeasured $\Omega_{b}^{*}$.

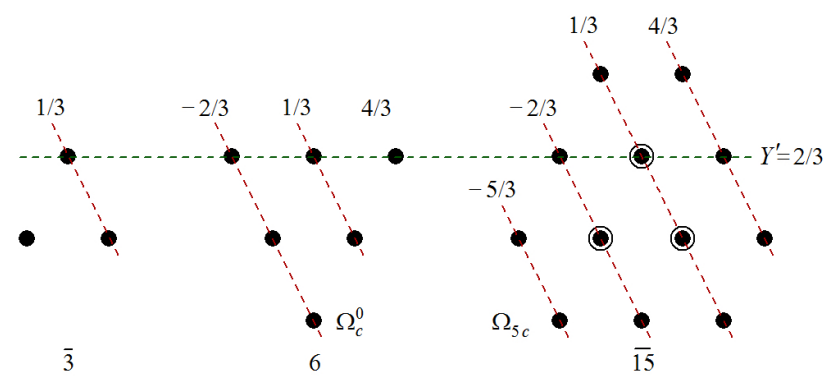

FIG. 2. Rotational band of a soliton with one valence quark stripped off. The soliton spin corresponds to the isospin $T^{\prime}$ of states on the quantization line $Y^{\prime}=2 / 3$. We show three lowest allowed representations: the antitriplet of spin 0 , the sextet of spin 1 and the lowest exotic representation $\overline{\mathbf{1 5}}$ of spin 1 or 0 . Diagonal lines indicate the states of equal charges (shown above the lines). Heavy quark charge has to be added.

\section{Exotic $\overline{\mathbf{1 5}}$ as a rotational excitation}

Analogously to the pentaquark $\overline{\mathbf{1 0}}$ representation, also in the present case, the soliton admits exotic representa- tions with the lowest one being $\overline{\mathbf{1 5}}$ (see Fig. 2). In this Section we study the properties of heavy pentaquarks constructed from a $\overline{\mathbf{1 5}}$ soliton and a heavy quark. Next possible exotic representation is $\overline{\mathbf{1 5}^{\prime}}=(p=0, q=4)$ with spin $J=1$, which however, is heavier than $\overline{\mathbf{1 5}}$.

As we can see from Fig. 2, the soliton in $\overline{\mathbf{1 5}}$ can be quantized both as spin $J=0$ and 1 (remember that the isospin of the states on $Y^{\prime}=2 / 3$ line corresponds to $\operatorname{spin}^{1}$.

In order to estimate the masses of the states in $\overline{\mathbf{1 5}}$ we shall use the general formula (1) with the rescaled moments of inertia $I_{1,2} \rightarrow \rho I_{1,2}$ :

$$
\begin{aligned}
& \mathcal{M}_{\overline{\mathbf{1 5}}, J=0}=M_{\text {sol }}+\frac{5}{2} \frac{1}{\rho I_{2}}, \\
& \mathcal{M}_{\overline{\mathbf{1 5}}, J=1}=M_{\text {sol }}+\frac{3}{2} \frac{1}{\rho I_{2}}+\frac{1}{\rho I_{1}} .
\end{aligned}
$$

Interestingly, the mass difference

$$
\Delta_{\overline{\mathbf{1 5}}}=\mathcal{M}_{\overline{\mathbf{1 5}}, J=0}-\mathcal{M}_{\overline{\mathbf{1 5}}, J=1}=\frac{1}{\rho}\left(\frac{1}{I_{2}}-\frac{1}{I_{1}}\right)
$$

is positive, since both in the model calculations and model-independent analyses, $I_{1} \sim 3 I_{2}$, which means counterintuitively - that the spin-1 soliton is lighter than the spin-0 one.

In order to estimate the masses of exotic heavy baryons it is useful to relate the mean $\overline{\mathbf{1 5}}$ mass to the mean $\mathbf{6}$ mass:

$$
\mathcal{M}_{\overline{\mathbf{1 5}}, J=1}=\mathcal{M}_{\mathbf{6}}+\frac{1}{\rho I_{2}}
$$

where we have from [2] $\mathcal{M}_{\mathbf{6}}=2580 \mathrm{MeV}$. Given rather large uncertainty of $I_{2}(8)$ and of the factor $\rho=1-0.66$ we get:

$$
\mathcal{M}_{\overline{\mathbf{1 5}}, J=1}=2980-3260 \mathrm{MeV} .
$$

Finally we have to calculate matrix elements of the symmetry-breaking Hamiltonian (3). The result reads:

$$
\begin{aligned}
\Delta_{s} M_{\overline{\mathbf{1 5}}} & =Y\left(\beta+\frac{17}{144}(\alpha-2 \gamma)\right) \\
& +\left(-\frac{2}{27}+\frac{1}{24}\left(T(T+1)-\frac{1}{4} Y^{2}\right)\right)(\alpha-2 \gamma)
\end{aligned}
$$

Note that in this case the $\delta$ term does not contribute. Using values from Eq.9 we obtain $\delta_{\Omega_{c}}=180 \mathrm{MeV}$, which should be further reduced by $11 \%$ giving $\delta_{\Omega_{c}}=160 \mathrm{MeV}$. We therefore predict that $\Omega_{c}$ from $\overline{\mathbf{1 5}}$ has mass in the range of $3140-3370 \mathrm{MeV}$ before the hyper-fine splitting, which we estimate using $\kappa / m_{c}=70 \mathrm{MeV}$ to be -50 and

\footnotetext{
1 From now on we use numerical values of the quantum numbers corresponding to $N_{c}=3$, which does not allow for proper $N_{c}$ counting.
} 
$+20 \mathrm{MeV}$ for spin $1 / 2$ and $3 / 2$ respectively. Therefore we see that these rough estimates indicate that some of the states seen by the LHCb might actually be exotic $\Omega_{5}$ c pentaquarks. At this point one should remember that these estimates are subject to the uncertainties due to the $\mathcal{O}\left(N_{c}^{0}\right)$ corrections discussed above.

The $\chi$ QSM allows to estimate the decay widths that proceed through the transition of the light sector associated with the emission of the pseudoscalar meson $\varphi$ $(=\pi, K, \eta)$. The heavy quark remains in the first approximation intact [3], and acts merely as a spectator. The transition operator can be expressed in terms of three coupling constants and the collective operators:

$$
\begin{aligned}
\mathcal{O}_{\varphi} & =3\left[G_{0} D_{\varphi i}^{(8)}-G_{1} d_{3 b c} D_{\varphi b}^{(8)} \hat{T}_{c}^{\prime}-G_{2} \frac{1}{\sqrt{3}} D_{\varphi 8}^{(8)} \hat{T}_{i}^{\prime}\right] \\
& \times \frac{p_{i}}{M_{1}+M_{2}} .
\end{aligned}
$$

In the present case we will have transitions $\overline{\mathbf{1 5}}_{1} \rightarrow \overline{\mathbf{3}}_{0}$ (where the lower index refers to $T^{\prime}=J$ ) that includes decays of exotic $\Omega_{c}$ measured by the LHCb, or $\overline{\mathbf{1 5}}_{1} \rightarrow \mathbf{6}_{1}$ that includes e.g. decays to $\Omega_{c}(2535)+\pi$ that have much larger phase space. Sandwiching operator 15 between rotational wave functions (4) one can calculate the effective decay constants

$$
\begin{array}{ll}
\overline{\mathbf{1 5}}_{1} \rightarrow \overline{\mathbf{3}}_{0} \quad G_{\overline{\mathbf{3}}}=G_{0}-\frac{1}{2} G_{1}, \\
\overline{\mathbf{1 5}}_{1} \rightarrow \mathbf{6}_{1} & G_{\mathbf{6}}=G_{0}-\frac{1}{2} G_{1}-G_{2} .
\end{array}
$$

In this normalization the pion-nucleon decay constant $\left(g_{\pi N N} \sim 13\right)$ reads

$$
g_{\pi N N}=\frac{7}{10}\left(G_{0}+\frac{1}{2} G_{1}+\frac{1}{14} G_{2}\right) .
$$

Interestingly, in the constituent quark limit [34, 35] of the $\chi \mathrm{QSM}$

$$
G_{0}=\left(N_{c}+2\right) G, G_{1}=4 G, G_{2}=2 G .
$$

In the present case, however, due to the fact that we have only $N_{c}-1$ occupied valence levels constant $G_{0}$ should be replaced

$$
G_{0} \rightarrow \bar{G}_{0}=\left(N_{c}+1\right) G .
$$

With this replacement $G_{\boldsymbol{6}}=0$, an effect similar to the nullification of the $\Theta^{+}$width in the same limit [35]. We therefore expect exotic $\overline{\mathbf{1 5}}_{1}$ pentaquarks to have small widths, even if $G_{\overline{3}} \neq 0$ in the constituent quark limit. We have cheked that for reasonable set of parameters $G_{0,1,2}$ one can indeed get the total decay width being of the order of $1 \mathrm{MeV}$.

In the present approach we cannot that easily calculate decay widths that include $D$ mesons. Fortunately the states that we discuss in this paper are lying below the threshold for such decays.

\section{Excited $\overline{3}$ and 6 multiplets as one-quark excitations}

Possible one quark excitations of the soliton depicted in Fig. 1.b have been discussed by Diakonov in Ref. 4]. By comparing possible excitation energies with the ones in the light sector, he has come to the conclusion that the most favourable transition that would lead to excited parity $(-)$ heavy baryons corresponds to the transition from a $K^{P}=1^{-}$sea level to an unoccupied $K^{P}=0^{+}$ state (see Fig. 1 c). Such a transition is not allowed in the light baryon sector. The very existence of a $K^{P}=1^{-}$ level as a sea level of the highest energy is of course a plausible conjecture that has to be confirmed by model calculations.

The first allowed $\mathrm{SU}(3)$ representation for one quark excited soliton is again $\overline{3}$ with $T^{\prime}=0$, which - according to (2) for $K=1-$ is quantized as spin 1. From (1) we have

$$
\mathcal{M}_{\overline{\mathbf{3}}}^{\prime}=M_{\mathrm{sol}}^{\prime}+\frac{1}{2 I_{2}}+\frac{a_{1}^{2}}{I_{1}}
$$

We will treat $\mathcal{M}_{\overline{3}}^{\prime}$ as a phenomenological parameter. Next possibility is flavor 6 with $T^{\prime}=1$, which may couple with $K=1$ to $J=0,1$ and 2. From (1) we have:

$$
\mathcal{M}_{\mathbf{6} J}^{\prime}=\mathcal{M}_{3}^{\prime}+\frac{1-a_{1}}{I_{1}}+\frac{a_{1}}{I_{1}} \times\left\{\begin{array}{rll}
-1 & \text { for } & J=0 \\
0 & \text { for } & J=1 \\
2 & \text { for } & J=2
\end{array} .\right.
$$

Both the $\overline{\mathbf{3}}$ and the $\mathbf{6}$ are subject to the $m_{s}$ splittings proportional to the hypercharge $Y$. For $\overline{3}$ the splitting parameter is given by the same formula as for the ground state antitriplet, and therefore we know its numerical value [2]:

$$
\delta_{\overline{\mathbf{3}}}^{\prime}=\frac{3}{8} \bar{\alpha}+\beta=\delta_{\overline{\mathbf{3}}}=-180 \mathrm{MeV} .
$$

In the case of the sextet the splittings depend on the soliton spin and read:

$$
\delta_{\mathbf{6} J}^{\prime}=\delta_{\mathbf{6}}-\frac{3}{20} \delta \times\left\{\begin{array}{r}
2 \text { for } J=0 \\
1 \text { for } J=1 \\
-1 \text { for } J=2
\end{array}\right.
$$

where $\delta_{\mathbf{6}}=-120 \mathrm{MeV}$ [2] corresponds to the ground state sextet splitting. Unfortunately, since we do not know the value of a new parameter $\delta$, we have no handle on the values of different $\delta_{6}^{\prime} J$.

Furthermore, according to Eq. (6), we have to include the hyper-fine splittings with, however, different chromomagnetic constant $\kappa^{\prime}$. The model predicts two $\mathrm{SU}(3)$ triplets of spin $1 / 2$ and $3 / 2$, two sextets of spin $1 / 2$ and $3 / 2$ and two sextets of spin $3 / 2$ and $5 / 2$ split by:

$$
\Delta_{\overline{\mathbf{3}}}^{\mathrm{hf}}=\Delta_{\mathbf{6} J=1}^{\mathrm{hf}}=\frac{\kappa^{\prime}}{m_{c}}, \quad \Delta_{\mathbf{6} J=2}^{\mathrm{hf}}=\frac{5}{3} \frac{\kappa^{\prime}}{m_{c}}
$$


and one sextet, presumably the lightest one, corresponding to $J=0$ with no hyper-fine splitting.

It is relatively easy to check the $\chi \mathrm{QSM}$ predictions for excited $\overline{\mathbf{3}}$, since there are rather well measured candidates. Indeed for $(1 / 2)^{-}$we have $\Lambda_{c}(2592)$ and $\Xi_{c}(2790)$ and for $(3 / 2)^{-}$there exist $\Lambda_{c}(2628)$ and $\Xi_{c}(2818)$. From this assignment we get $\delta_{\overline{3}}^{\prime}=-198 \mathrm{MeV}$ and $-190 \mathrm{MeV}$ respectively, in relative good agreement with Eq.21.). Furthermore, we can extract two other parameters:

$$
\begin{aligned}
\frac{\kappa^{\prime}}{m_{c}} & =\frac{1}{3}\left(M_{\Lambda_{c}(2628)}+2 M_{\Xi_{c}(2818)}\right) \\
& -\frac{1}{3}\left(M_{\Lambda_{c}(2252)}+2 M_{\Xi_{c}(2790)}\right)=30 \mathrm{MeV} \\
\mathcal{M}_{\overline{3}}^{\prime} & =\frac{2}{9}\left(M_{\Lambda_{c}(2628)}+2 M_{\Xi_{c}(2818)}\right) \\
& +\frac{1}{9}\left(M_{\Lambda_{c}(2252)}+2 M_{\Xi_{c}(2790)}\right)=2744 \mathrm{MeV}
\end{aligned}
$$

In the next Section we shall discuss phenomenological application of the $\chi$ QSM to the charmed sextet.

\section{POSSIBLE INTERPRETATIONS OF THE LHCB $\Omega_{c}$ STATES}

The natural scenario, which we will follow in this analysis, is that higher spin states (or more precisely higher $J$ states) become heavier as the spin increases. This as- sumption leads then to a $\mathbf{6}$ spectrum depicted schematically in Fig. 3 with $\left(J=0,1 / 2^{-}\right)$state corresponding to $\Omega_{c}(3000)$. This spectrum has to be supplemented by two possible states $1 / 2^{+}$and $3 / 2^{+}$belonging to the exotic $\overline{15}$.

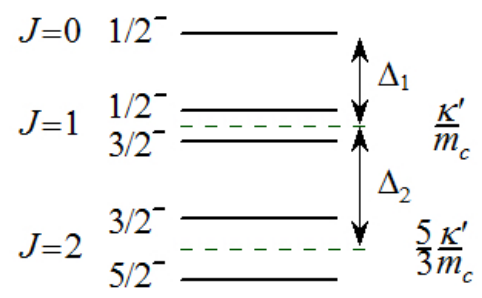

FIG. 3. Schematic spectrum of excited sextets.

The splittings $\Delta_{1,2}$ in Fig. 3 correspond to the $\Omega_{c}$ masses for given $J$ before the hyper-fine splitting and read:

$$
\Delta_{1}=\frac{a_{1}}{I_{1}}+\frac{3}{20} \delta, \quad \Delta_{2}=2 \Delta_{1}
$$

The $\chi$ QSM predicts five $\Omega_{c}$ states belonging to the excited sextet. Therefore we may try to identify all five $\mathrm{LCHb}$ resonances with these states. The corresponding scenario is summarized in Table I] We see that in this scenario the relation between the mass splittings $(26)$ is badly broken. This can be further illustrated by observing that the $\Omega_{c}$ masses satisfy in the $\chi$ QSM two orthogonal sum rules $\sigma_{1}=\sigma_{2}=0$ with:

$$
\begin{aligned}
& \sigma_{1}=6 \Omega_{c}\left(J=0,1 / 2^{-}\right)-\Omega_{c}\left(J=1,1 / 2^{-}\right)-8 \Omega_{c}\left(J=1,3 / 2^{-}\right)+3 \Omega_{c}\left(J=2,5 / 2^{-}\right), \\
& \sigma_{2}=-4 \Omega_{c}\left(J=0,1 / 2^{-}\right)+9 \Omega_{c}\left(J=1,1 / 2^{-}\right)-3 \Omega_{c}\left(J=1,3 / 2^{-}\right)-5 \Omega_{c}\left(J=2,3 / 2^{-}\right)+3 \Omega_{c}\left(J=2,5 / 2^{-}\right),
\end{aligned}
$$

which are numerically badly violated by the assignment of the minimal scenario. Let us mention the authors of Ref. [12, who try to interpret the LHCb states within the quark-diquark model, came to the similar conclusion.

As can be seen from Table I the parameter for the hyper-fine splitting deviates considerably from that determined from the experimental data for the excited $\overline{\mathbf{3}}^{\prime}$ given in Eq. (24). Also in this scenario the widths of the would be hyper-fine split partners are very different. All these arguments suggest that such minimal scenario is not realistic in the mean-field picture of baryons.

Given that the minimal scenario does not work, we may try to attribute some of the five narrow LHCb $\Omega_{c}$ 's to possible exotic $\overline{\mathbf{1 5}}$ multiplet which naturally emerges in our picture. The states $\Omega_{c}(3050)$ and $\Omega_{c}(3119)$ are good candidates for the $1 / 2^{+}$and $3 / 2^{+}$hyper-fine split $\Omega_{c}$ members of the $\overline{\mathbf{1 5}}$. Firstly, the corresponding hyperfine splitting parameter $\kappa / m_{c} \approx 70 \mathrm{MeV}$, in excellent agreement with the same parameter determined from the data on the ground-state sextet [2]. Secondly, the widths of $\Omega_{c}(3050)$ and $\Omega_{c}(3119)$ are of order $1 \mathrm{MeV}$ in agreement with our expectations discussed in Sect. III Cabove. The assignment of the $\mathrm{LHCb}$ states in this scenario is summarized in Table II. We see that in this scenario the excited sextet states with $J=2$ have masses above the $\Xi+D$ threshold at $3185 \mathrm{MeV}$, i.e. they can have rather large widths and are not clearly seen in the LHCb data.

We have tried several other possibilities to distribute the observed states over the negative parity excited sextet and the positive parity $\overline{\mathbf{1 5}}$, however all of them give less consistent picture.

One can check the suggested identification of new $\Omega_{c}$ states in various ways. The simplest one would be to search for the isospin partners of $\Omega_{c}^{0}$ from the $\overline{\mathbf{1 5}}$. For example, they can be searched in mass distribution of $\Xi_{c}^{0}+K^{-}$or $\Xi_{c}^{+}+\bar{K}^{0}$, the $\Omega_{c}^{0}$ 's from the sextet do not decay into these channels. Another possibility is to search for the other exotic members of the $\overline{\mathbf{1 5}}$ especially the lightest 


\begin{tabular}{|c|c|c|c|c|}
\hline$J$ & $S^{P}$ & $M[\mathrm{MeV}]$ & $\kappa^{\prime} / m_{c}[\mathrm{MeV}]$ & $\Delta_{J}[\mathrm{MeV}]$ \\
\hline 0 & $\frac{1}{2}^{-}$ & 3000 & - & - \\
\hline \multirow{2}{*}{1} & $\frac{1}{2}^{-}$ & 3050 & 16 & 61 \\
& $\frac{3}{2}^{-}$ & 3066 & & \\
\hline \multirow{2}{*}{2} & $\frac{3}{2}^{-}$ & 3090 & 17 & 47 \\
\hline & $\frac{5}{2}^{-}$ & 3119 & & \\
\hline
\end{tabular}

TABLE I. Scenario 1. All LHCb $\Omega_{c}$ states are assigned to the excited sextets. This assignment requires hyperfine splitting which is almost two times smaller than in the $\overline{\mathbf{3}}$ case and relation 26 is badly broken.

$B_{c}$-baryons (see the next Section).

\begin{tabular}{|c|l|c|c|c|}
\hline$J$ & $S^{P}$ & $M[\mathrm{MeV}]$ & $\kappa^{\prime} / m_{c}[\mathrm{MeV}]$ & $\Delta_{J}[\mathrm{MeV}]$ \\
\hline 0 & $\frac{1}{2}^{-}$ & 3000 & - & - \\
\hline \multirow{2}{*}{1} & $\frac{1}{2}^{-}$ & 3066 & 24 & 82 \\
& $\frac{3}{2}^{-}$ & 3090 & & \\
\hline \multirow{2}{*}{2} & $\frac{3}{2}^{-}$ & 3222 & input & input \\
& $\frac{5}{2}^{-}$ & 3262 & 24 & 164 \\
\hline
\end{tabular}

TABLE II. Scenario 2. Only three LHCb states are assigned to the sextets. Using relations $(26)$ and 23 we calculate the masses of $J=2$ states (marked in italics) that fall into a large bump seen by the LHCb above $3.2 \mathrm{GeV}$. In this scenario two narrow states $\Omega_{c}(3050)$ and $\Omega_{c}(3119)$ are interpreted as exotic $\overline{\mathbf{1 5}}$ pentaquarks.

\section{MORE ON EXOTIC $\overline{\mathbf{1 5}}$}

\section{A. Partners of $\Omega_{c}(3050)$ and $\Omega_{c}(3119)$}

In the previous Section we have demonstrated that the favourable scenario is to identify the observed narrow resonances $\Omega_{c}(3050)$ and $\Omega_{c}(3119)$ as the $1 / 2^{+}$and $3 / 2^{+}$ members of the exotic $\overline{\mathbf{1 5}}$ multiplet. Now with the help of the mass formula (16) we can predict the masses of other members of the exotic $\overline{\mathbf{1 5}}$. The parameters of the mass formula $(\alpha, \beta, \gamma)$ are fixed by the spectrum of the ground-state light multiplets $(9)$. Note that the spectrum has to be calculated using rescaled $\alpha \rightarrow \bar{\alpha}=2 / 3 \alpha$. Furthermore the splittings have to be reduced by $11 \%$ to account for the effect discussed in Sect. IIIB The predicted masses 2 of $\overline{\mathbf{1 5}}$ are summarized in Table III

\footnotetext{
2 Note that the predicted masses can be affected by the mixing of non-exotic members of the $\overline{\mathbf{1 5}}$ with the ground-state and excited $\overline{\mathbf{3}}$ and $\mathbf{6}$, similarily how it happens in the light baryon sector, see Ref. 27.

${ }^{3}$ We adopt the naming scheme suggested by D.I. Diakonov [4]
}

that with these numbers we get $\mathcal{M}_{\overline{\mathbf{1 5}}, J=1}=2935 \mathrm{MeV}$, just a little below lower limit of Eq. [13].

\begin{tabular}{|l|r|c|c|c|}
\hline & $Y$ & $T$ & $S^{P}=\frac{1}{2}^{+}$ & $S^{P}=\frac{3}{2}^{+}$ \\
\hline$B_{c}$ & $\frac{5}{3}$ & $\frac{1}{2}$ & 2685 & 2754 \\
\hline$\Sigma_{c}$ & $\frac{2}{3}$ & 1 & 2808 & 2877 \\
\hline$\Lambda_{c}$ & $\frac{2}{3}$ & 0 & 2806 & 2875 \\
\hline$\Xi_{c}$ & $-\frac{1}{3}$ & $\frac{1}{2}$ & 2928 & 2997 \\
\hline$\Xi_{c}^{3 / 2}$ & $-\frac{1}{3}$ & $\frac{3}{2}$ & 2931 & 3000 \\
\hline$\Omega_{c}$ & $-\frac{4}{3}$ & 1 & 3050 & 3119 \\
\hline
\end{tabular}

TABLE III. Predicted masses (in $\mathrm{MeV}$ ) of $1 / 2^{+}$and $3 / 2^{+}$ $\overline{15}$-plet under the assumption that $\Omega_{c}$ members are identified with the observed $\Omega_{c}(3050)$ and $\Omega_{c}(3119)$.

The exotic $\overline{15}$-plet contains six explicitly exotic states: $B_{c}^{+}, B_{c}^{++}$(with the minimal quark content $c u d d \bar{s}$ and cuud $\bar{s}), \quad \Xi_{c}^{3 / 2-}, \Xi_{c}^{3 / 2++}(c d d s \bar{u}, \quad$ cuus $\bar{d})$, and $\Omega_{c}^{-}, \Omega_{c}^{+}$ $(c d s s \bar{u}, c u s s \bar{d})$. The detailed properties of the $\overline{\mathbf{1 5}}$-plet we shall study elsewhere. Here we note that the predicted mass of the lightest $\overline{\mathbf{1 5}}$ member, the $B_{c}$-baryon, lies slightly below the strong decay threshold to $\left(\Lambda_{c}, \Sigma_{c}\right)+K$, hence we predict that the $B_{c}$-baryon decays only weakly.

The $\overline{\mathbf{1 5}}$-plet was discussed for the first time by D. I. Diakonov in Ref. 4]. In this paper the $\overline{\mathbf{1 5}}$-plet was obtained due to a specific quark transition between quark levels in the mean-field (an analog of the Gamow-Teller transition), so the picture there is different from ours. In Ref. 4 the $\overline{\mathbf{1 5}}$-plet is considerably lighter than in our picture. For example, the mass of the $B_{c}$ baryon is $2420 \mathrm{MeV}$. We shall compare in detail the two pictures elsewhere.

\section{B. On excited $\Omega_{b}$}

The mean-field picture of baryons presented here can be easily generalized to baryons with a bottom quark. The main feature of our approach is that the mean field does not depend on the heavy quark mass. So, if we replace the charm quark by the bottom one, we have to make an overall shift of the masses and rescale the hyperfine splittings.

As for the overall shift of the masses, we take the mass difference of the ground-state antitriplets for charmed and bottom baryons:

$$
M \frac{b}{\mathbf{3}}-M \frac{c}{\mathbf{3}}=3327 \mathrm{MeV},
$$

which was determined in Ref. 2, where we have also demonstrated that the ratio of the hyper-fine mass splittings in the charm and bottom ground-state sextets is close to $\sim 0.3$, being in excellent agreement with the mass ratio $m_{c} / m_{b}$.

Performing the overall mass shift and rescaling the hyper-fine splittings, we obtain the following prediction for the excited $\Omega_{b}: \Omega_{b}\left(6327,1 / 2^{-}\right), \Omega_{b}\left(6404,1 / 2^{-}\right)$, 
$\Omega_{b}\left(6411,3 / 2^{-}\right), \Omega_{b}\left(6566,3 / 2^{-}\right)$and $\Omega_{b}\left(6578,5 / 2^{-}\right)$belonging to the excited sextets, and $\Omega_{b}\left(6409,1 / 2^{+}\right)$and $\Omega_{b}\left(6430,3 / 2^{+}\right)$belonging to the exotic $\overline{\mathbf{1 5}}$-plet.

\section{SUMMARY AND CONCLUSIONS}

The goal of the present paper was to classify the $\Omega_{c}$ baryons that have been recently reported by the $\mathrm{LHCb}$ collaboration [1], employing the mean-field approach. The mean-field picture of baryons, being justified by the large- $N_{c}$ limit, offers a unified description of light and heavy baryons. We have shown in Ref. 2] that the universal mean field gives simultaneously good description of the ground-state $\overline{\mathbf{3}}$ and $\mathbf{6}$ multiplets of heavy baryons. Also the ground-state light baryon multiplets are well described [6]. In the present work we have demonstrated that the same picture predicts the following excited states for heavy-quark baryons in the mass region of $3000-3200 \mathrm{MeV}$ :

- two hyper-fine split $\left(1 / 2^{-}\right.$and $\left.3 / 2^{-}\right) \overline{\mathbf{3}}^{\prime}$ which experimentally have very good candidates,

- five excited sexstets (rotationally and hyper-fine split) with quantum numbers $\left(J=0,1 / 2^{-}\right),(J=$ $\left.1,1 / 2^{-}, 3 / 2^{-}\right)$and $\left(J=2,3 / 2^{-}, 5 / 2^{-}\right)$, where $J$ denotes the soliton spin,

- two hyper-fine split exotic $\overline{\mathbf{1 5}}$-plets with quantum numbers $1 / 2^{+}$and $3 / 2^{+}$.

Due to the universality of our mean field picture the basic properties of these excitations are fixed by light baryons and by ground-state multiplets of heavy quark baryons. The predictions for the excited $\overline{\mathbf{3}}^{\prime}$-plets are in excellent agreement with the experimental spectrum of the excited $\Lambda_{c}$ and $\Xi_{c}$.

The observation of the new excited $\Omega_{c}^{0}$ 's allows us to get insight into the excited sextets and $\overline{\mathbf{1 5}}$ plets. We identify the observed $\Omega_{c}(3000), \Omega_{c}(3066)$ and $\Omega_{c}(3090)$ with $\left(J=0,1 / 2^{-}\right)$and $\left(J=1,1 / 2^{-}, 3 / 2^{-}\right)$ states from the excited sextet, whereas we identify the most narrow $\Omega_{c}(3050)$ and $\Omega_{c}(3119)$ states with the $\left(J=1,1 / 2^{+}, 3 / 2^{+}\right)$states from the exotic $\overline{\mathbf{1 5}}$ multiplet. The remaining two $\left(J=2,3 / 2^{-}, 5 / 2^{-}\right)$states from the sextet have masses above $\Xi+D$ threshold $(3185 \mathrm{MeV})$, so they are probably hidden in a large bump observed by the LHCb collaboration above $3200 \mathrm{MeV}$. It should be stressed that the simplest scenario in which all five LHCb $\Omega_{c}^{0}$ states are classified as members of the excited sextets, contradicts general mass formulae derived within the $\chi \mathrm{QSM}$.

The simplest way to falsify our identification is to search for the isospin partners of $\Omega_{c}^{0}$ from the $\overline{\mathbf{1 5}}$. For example, they can be searched in the mass distribution of $\Xi_{c}^{0}+K^{-}$or $\Xi_{c}^{+}+\bar{K}^{0}$, the $\Omega_{c}^{0}$ 's from the sextet do not decay into these channels.

\section{ACKNOWLEDGMENTS}

The authors are grateful to Victor Petrov for illuminating discussions. H.-Ch. K is grateful to J.Y. Kim and Y.S. Jun for discussion. The work of H.-Ch.K. was supported by Basic Science Research Program through the National Research Foundation of Korea funded by the Ministry of Education, Science and Technology (Grant Number: NRF-2015R1A2A2A04007048). MVP is thankful to Department of Theoretical Physics of Irkutsk State University for kind hospitality and for the support. The work of MVP is supported by CRC110.
[1] R. Aaij et al. [LHCb Collaboration], arXiv:1703.04639 [hep-ex].

[2] G. S. Yang, H.-Ch. Kim, M. V. Polyakov and M. Praszałowicz, Phys. Rev. D 94, 071502 (2016).

[3] N. Isgur and M. B. Wise, Phys. Lett. B 232, 113 (1989) and Phys. Rev. Lett. 66, 1130 (1991).

[4] D. Diakonov, arXiv:1003.2157 [hep-ph].

[5] D. Diakonov, V. Petrov and A. A. Vladimirov, Phys. Rev. D 88, 074030 (2013)

[6] V. Petrov, Acta Phys. Polon. B 47, 59 (2016).

[7] Z. G. Wang, arXiv:1704.01854 [hep-ph].

[8] H. X. Chen, Q. Mao, W. Chen, A. Hosaka, X. Liu and S. L. Zhu, arXiv:1703.07703 [hep-ph].

[9] S. S. Agaev, K. Azizi and H. Sundu, arXiv:1703.07091 [hep-ph].

[10] K. L. Wang, L. Y. Xiao, X. H. Zhong and Q. Zhao, arXiv:1703.09130 [hep-ph].

[11] M. Padmanath and N. Mathur, arXiv:1704.00259 [hep$\mathrm{ph}]$.

[12] M. Karliner and J. L. Rosner, arXiv:1703.07774 [hep-ph].

[13] W. Wang and R. L. Zhu, arXiv:1704.00179 [hep-ph].
[14] H. Y. Cheng and C. W. Chiang, arXiv:1704.00396 [hep$\mathrm{ph}]$.

[15] H. Huang, J. Ping and F. Wang, arXiv:1704.01421 [hep$\mathrm{ph}]$.

[16] G. Yang and J. Ping, arXiv:1703.08845 [hep-ph].

[17] E. Witten, Nucl. Phys. B 160, 57 (1979) and Nucl. Phys. B 223, 422 (1983) and Nucl. Phys. B 223, 433 (1983).

[18] C. V. Christov, A. Blotz, H.-Ch. Kim, P. Pobylitsa, T. Watabe, T. Meissner, E. Ruiz Arriola and K. Goeke, Prog. Part. Nucl. Phys. 37, 91 (1996) hep-ph/9604441.

[19] G. S. Adkins and C. R. Nappi, Nucl. Phys. B 249, 507 (1985).

[20] B. Moussallam and D. Kalafatis, Phys. Lett. B 272, 196 (1991). doi:10.1016/0370-2693(91)91819-H

[21] N. N. Scoccola and H. Walliser, Phys. Rev. D 58, 094037 (1998) hep-ph/9805340.

[22] H. Walliser, Phys. Lett. B 432, 15 (1998) hep$\mathrm{ph} / 9710232$.

[23] E. N. Nikolov, W. Broniowski, C. V. Christov, G. Ripka and K. Goeke, Nucl. Phys. A 608, 411 (1996) hep$\mathrm{ph} / 9602274$. 
[24] K. Goeke, M. M. Musakhanov and M. Siddikov, Phys. Rev. D 76, 076007 (2007) arXiv:0707.1997 [hep-ph]].

[25] K. Goeke, H.-Ch. Kim, M. M. Musakhanov and M. Siddikov, Phys. Rev. D 76, 116007 (2007) arXiv:0708.3526 [hep-ph]].

[26] G. S. Yang and H.-Ch. Kim, Prog. Theor. Phys. 128, 397 (2012) arXiv:1010.3792 [hep-ph]].

[27] K. Goeke, M. V. Polyakov and M. Praszałowicz, Acta Phys. Polon. B 42, 61 (2011) arXiv:0912.0469 [hep-ph]].

[28] T. Nakano et al. [LEPS Collaboration], Phys. Rev. Lett. 91, 012002 (2003) hep-ex/0301020.
[29] T. Nakano et al. [LEPS Collaboration], Phys. Rev. C 79, 025210 (2009) arXiv:0812.1035 [nucl-ex]].

[30] V. V. Barmin et al. [DIANA Collaboration], Phys. Rev. C 89, no. 4, 045204 (2014) arXiv:1307.1653 [nucl-ex]].

[31] M. J. Amaryan et al., Phys. Rev. C 85, 035209 (2012) arXiv:1110.3325 [hep-ex]].

[32] M. Anghinolfi et al. [CLAS Collaboration], arXiv:1204.1105 [hep-ex].

[33] C. Alt et al. [NA49 Collaboration], Phys. Rev. Lett. 92, 042003 (2004) hep-ex/0310014.

[34] M. Praszałowicz, A. Blotz and K. Goeke, Phys. Lett. B 354, 415 (1995) hep-ph/9505328.

[35] D. Diakonov, V. Petrov and M. V. Polyakov, Z. Phys. A 359, 305 (1997) hep-ph/9703373. 